PROBLEMS

OF MANAGEMENT

IN THE $21^{\text {st }}$ CENTURY

Volume 1, 2011

60

\title{
AN EXPONENTIAL UTILITY APPROACH TO EIGENVECTOR METHOD IN THE ANALYTIC HIERARCHY PROCESS: AN IDEA INTRO
}

\author{
Pawel Tadeusz Kazibudzki \\ Jan Dlugosz University in Czestochowa, Poland \\ E-mail: emailpoczta@gmail.com
}

\begin{abstract}
It is crucial for a credible decision making theory to provide unique answers for the alternatives of a decision. However, different methods and algorithms devised in order to elicit true priority vectors from intuitive judgments give different priority vectors, especially when judgments are inconsistent what constantly takes place in the real life. One could deduce that such variety of results that a potential decision maker can obtain violates the uniqueness requirement mentioned above and therefore is seemed unacceptable. On the other hand, it is known that Eigenvalue Method, commonly applied in the Analytic Hierarchy Process, captures transitivity in matrices that are not consistent in the unique way. That could lead to a conclusion that maybe the Eigenvalue Method is necessary and sufficient to facilitate credible decision making based on priority weighting followed by inconsistent matrices comprising pairwise comparison judgments. However, the Eigenvalue Method, despite of obvious advantages, has also few disadvantages that cannot be neglected. A research described in this article gives rise to a new method for priority vectors deriving which coincides with the Eigenvalue Method but avoids its drawbacks.

Key words: Analytic Hierarchy Process, eigenvalue method, deriving priority vector, optimization models, condition of order preservation.
\end{abstract}

\section{Introduction}

Managers are struggling with problems demanding decision making not since nowadays, it is a fact. It is also a fact that science deals with explanation and modeling of decisional problems for a very long time. The most important areas of research might be distinguished taking into consideration a degree of accuracy with problem specification.

If one knows exactly what options one has and what utility is entailed with one's choice, a decision making procedure which leads to pick the best possible decision is to look for a maximum of utility function. This kind of problems constitutes the area of interest for a mathematical optimization theory. If, on the other hand, some information associated with a certain decisional problem is not determined but rather random, then a decision making procedure that leads to pick the best possible decision is to look for a maximum of utility function expected value. This kind of problems examines a discipline called probability theory. If one is dealing only with some degree of uncertainty then a mathematical theory of decision suggests different strategies that lead to a rational decision, for example mini-max theory provides a highest utility in worst conditions. If however uncertainty is associated with many elements of decisional situation, for example: decision utility, decision meaning, criteria significance, etc., then one may apply methods based on fuzzy sets theory.

There are also AHP based theories that deal with priorities recognition and their 
establishment on the bases of intuitive judgments. They are designed in order to facilitate a decision making process either and plenty of them devised for this purpose can be found in $\mid \begin{aligned} & \text { PROBLEMS } \\ & \text { OF MANAGEMENT } \\ & \text { IN THE 21st CENTURY } \\ & \text { Volume 1, } 2011\end{aligned}$ the literature. Some of them are based on different statistical concepts (Basak, 1998; Crawford et. al., 1985; Lipovetsky et. al., 1997), while others focus on constrained optimization models (Bryson, 1995; Cook et. al., 1988; Crawford et. al., 1985; Hashimoto, 1994; Lin, 2006; Sun et. al., 2006). Obviously every method proposed in the literature has its own pros and cons debate and thus one can find supporters and adversaries for each of them.

\section{Recognition of a Problem's Background}

Comparative studies of different prioritization methods (Basak, 1998; Budescu et. al., 1986; Dong et. al., 2008; Fichtner, 1986; Hovanov et. al., 2008; Saaty et. al., 1998; Saaty et. al., 1984), as well as suggestions to blend various prioritization techniques for better true priority vector estimates (Srdjevic, 2005), can be found as well. It seems however that most of known prioritization methods can be numbered among constrained optimization ones (Choo et. al., 2004).

These methods can be described in the following manner. Let it be presumed that one have only judgments (estimates) of the relative weights of a set of activities. Then one can express them in a pairwise comparison matrix (PCM) denoted as $\boldsymbol{A}$ with elements $a_{i j}=a_{i} a_{j}$ that can be presented as follows:

$$
A=\left[\begin{array}{ccccc}
a 1 / a 1 & a 1 / a 2 & a 1 / a 3 & \ldots & a 1 / a n \\
a 2 / a 1 & a 2 / a 2 & a 2 / a 3 & \ldots & a 2 / a n \\
a 3 / a 1 & a 3 / a 2 & a 3 / a 3 & \ldots & a 3 / a n \\
\vdots & \vdots & \vdots & & \vdots \\
a n / a 1 & a n / a 2 & a n / a 3 & \ldots & a n / a n
\end{array} \mid\right.
$$

Also, let it be denoted $\boldsymbol{A}(\boldsymbol{w})$ as the symbol of a matrix with elements $w_{i j}=w_{i} / w_{j}$ that can be presented as follows:

$$
A(w)=\left[\begin{array}{ccccc}
w 1 / w 1 & w 1 / w 2 & w 1 / w 3 & \ldots & w 1 / w n \\
w 2 / w 1 & w 2 / w 2 & w 2 / w 3 & \ldots & w 2 / w n \\
w 3 / w 1 & w 3 / w 2 & w 3 / w 3 & \ldots & w 3 / w n \\
\vdots & \vdots & \vdots & & \vdots \\
w n / w 1 & w n / w 2 & w n / w 3 & \ldots & w n / w n
\end{array}\right]
$$

Now, if one would like to recover the vector of weights $w=\left[w_{1}, w_{2}, w_{3}, \ldots, w_{n}\right]^{T}$ which true relative weights of a set of activities can be created from, as in the case of above matrix $\boldsymbol{A}(\boldsymbol{w})$, one can apply some optimization method which seeks a vector $\boldsymbol{w}$ as a solution of the following minimization problem:

$$
\min D(A, A(w)
$$


subject to some assigned constraints such as for instance positive coefficients and normalization condition. As the distance function $\boldsymbol{D}$ measures an interval between matrices $\boldsymbol{A}$ and $\boldsymbol{A}(\boldsymbol{w})$, various ways of its definition lead to different prioritization concepts. It seems that most popular one is called the logarithmic least squares method (LLSM), known also as geometric mean method (Crawford, 1987).

However, there is a method that cannot be recognized as one of these characterized as constrained optimization ones. This is also the first and most commonly used prioritization method which is also a fundamental part of a mathematical theory for deriving ratio scale priority vectors $(\mathrm{PV})$ from positive reciprocal matrices with entries set on the bases of pairwise comparisons. A theory is called the Analytic Hierarchy Process (AHP) and it uses a principal Eigenvalue Method (EM) to derive priority vectors (Saaty, 1994). It can be described in the following manner. Let it be presumed that one knows the relative weights of a set of activities. Then one can express them in a PCM like $\boldsymbol{A}(\boldsymbol{w})$ which was described above. Now, if one would like to recover the vector of weights $\boldsymbol{w}$ which the ratios in $\boldsymbol{A}(\boldsymbol{w})$ can be created from, one could take the matrix product of the matrix $A(w)=\left[w_{i j}\right]_{\mathrm{nxn}}$ with the vector $\boldsymbol{w}$ in order to receive:

$\left[\begin{array}{ccccc}w 1 / w 1 & w 1 / w 2 & w 1 / w 3 & \ldots & w 1 / w n \\ w 2 / w 1 & w 2 / w 2 & w 2 / w 3 & \ldots & w 2 / w n \\ w 3 / w 1 & w 3 / w 2 & w 3 / w 3 & \ldots & w 3 / w n \\ \vdots & \vdots & \vdots & & \vdots \\ w n / w 1 & w n / w 2 & w n / w 3 & \ldots & w n / w n\end{array}\right] \times\left[\begin{array}{c}w 1 \\ w 2 \\ w 3 \\ \vdots \\ w n\end{array}\right]=\left[\begin{array}{c}n w 1 \\ n w 2 \\ n w 3 \\ \vdots \\ n w n\end{array}\right]$

If one knows $\boldsymbol{a}(\boldsymbol{w})$, but not $\boldsymbol{w}$, one can solve this problem for $\boldsymbol{w}$. Solving for a nonzero solution to this set of equations is very common procedure and is known as an eigenvalue problem:

$$
A(w) \times w=l a m b d a \times w
$$

In order to find the solution to this set of equations, in general, one need to solve an $n$th order equation for lambda that, in general, leads to $n$ unique values for lambda, with an associated vector $\boldsymbol{w}$ for each of the $n$ values. However in the case of PCM based on priority weighting, the matrix $\boldsymbol{A}(\boldsymbol{w})$ has a special form, since each row is a constant multiple of the first row. In this case a matrix $\boldsymbol{A}(\boldsymbol{w})$ has only one nonzero eigenvalue and since the sum of the eigenvalues of a positive matrix is equal to the sum of its diagonal elements, the only nonzero eigenvalue in such a case equals the size of the matrix and can be denoted as $l a m b d a_{\max }=n$. If the elements of a matrix $\boldsymbol{A}(\boldsymbol{w})$ satisfy the condition $w_{i j}=1 / w_{j i}$ for all $i, j=1, \ldots, n$ then the matrix $\boldsymbol{A}(\boldsymbol{w})$ is said to be reciprocal. If its elements satisfy the condition $w_{i k} w_{k j}=w_{i j}$ for all $i, j, k=1, \ldots$, $n$ and the matrix is reciprocal, then it is called consistent. Finally, the matrix $\boldsymbol{A}(\boldsymbol{w})$ is said to be transitive if the following condition holds: if an element $w_{i j}$ is not less than an element $w_{i k}$ then $w_{i j} \geq w_{k}$ for $i=1, \ldots, \mathrm{n}$. Now again, it is obvious that in the real life during priority weighting one does not have $\boldsymbol{A}(\boldsymbol{w})$ but only its estimate $\boldsymbol{A}$ containing one's intuitive judgments, more or 


\author{
PROBLEMS \\ OF MANAGEMENT \\ IN THE $21^{\text {st }}$ CENTURY \\ Volume 1, 2011
}

less close to $\boldsymbol{A}(\boldsymbol{w})$ in accordance with one's skills, experience, etc. In such a case consistency property obviously does not hold and the relation between elements of $\boldsymbol{A}$ and $\boldsymbol{A}(\boldsymbol{w})$ can be expressed in the following form:

$$
a_{i j}=e_{i j} w_{i j}
$$

where $e_{i j}$ is a perturbation factor which should be close to 1 . It has been shown that for any matrix small perturbations in the entries imply similar perturbations in the eigenvalues, that is why in order to estimate the true priority vector $\boldsymbol{w}$ one need to solve the following matrix equation:

$$
A \times w=l a m b d a_{\max } \times w
$$

where $l a m b d a_{\text {max }}$ is the principal eigenvalue, it is not smaller than $n$, and other lambdas are close to zero. The estimates of the true priority vector $\boldsymbol{w}$ can be found then by normalizing the eigenvector corresponding to the largest eigenvalue in equation [7] which is simple and its existence is guaranteed by Perron's Theorem (Saaty, 1994) In practice the EM solution is obtained by raising the matrix $\boldsymbol{A}$ to a sufficiently large power, then summing over the rows and normalizing in order to receive $\boldsymbol{w}$. This concept can be also delivered in a form of the following formula:

where $e=[1,1, \ldots, 1]$.

$$
w=\lim _{k \rightarrow \infty}\left(\frac{A^{k} \times e^{T}}{e \times A^{k} \times e^{T}}\right)
$$

\title{
Definition of a Problem
}

It is prerequisite that an authentic decision making theory should provide unique answers for the alternatives of a decision. As it was presented above different methods and algorithms were devised in order to elicit true priority vectors from intuitive judgments. When judgments are rather consistent, the results of all approaches rather coincide. However, in the real life, judgments are constantly inconsistent. Such a situation gives rise to different priority vectors due to application of different methods. It was also proved that especially in multicriteria processes even when different methods provide priority vectors that are close, both on criteria and alternatives, after synthesis according to a well-prescribed procedure (Saaty, 1980) (standard AHP aggregation based on weighting and adding) the rank order of the alternatives can vary (Saaty, 1998). One could deduce that such variety of results that a potential decision maker can obtain violates the uniqueness requirement mentioned above and therefore is seemed unacceptable. On the other hand, it is known that EM captures transitivity in matrices that are not consistent in the unique way. That could lead to a conclusion that maybe the EM is necessary and sufficient to facilitate credible decision making based on priority weighting followed by inconsistent matrices comprising pairwise comparison judgments. However, let it be remembered that EM, despite of obvious advantages, has also few disadvantages and drawbacks. First of all it requires complex calculations connected with an iterative procedure given by the equation [8]. Secondly, it enforces reciprocity of the PCM through an imposed convention concerning the PCM inputs collection. Typically the PCM inputs are gathered only for the elements placed above diagonal elements of the matrix $\boldsymbol{A}$. The remaining ones are entered as the inverse of the corresponding symmetric elements in relation to diagonal elements of the matrix $\boldsymbol{A}$. It is crucial to notice that such a kind of consistency imposition loses additional information which could be revealed during data collection for the lower triangle of $\boldsymbol{A}$ and in consequence may lead to worse estimates of PV. For example, if one is supposed to judge if one likes Helen three times more than Betty, and one seems one does, it does not necessarily have 
PROBLEMS

OF MANAGEMENT

IN THE $21^{\text {st }}$ CENTURY

Volume 1, 2011

64 to mean that in comparison to Helen, one's judgment will be that one likes Betty three times less. This example only underlines a nature of intuitive judgments which from a definition are not a perfect measurement device as they are based on the human perception. Finally, what was noticed quite recently (Bana e Costa et. al., 2008), the EM does not satisfy a condition of order preservation (COP) what means that PVs derived on the bases of EM do not preserve the intensity of preferences.

\section{Conception of a Problem Solution}

It has been already deduced (Grzybowski, 2010) that instead of solving the eigenvalue equation [7] one may seek a vector $\boldsymbol{w}$ which best estimates the equation [5]. In order to satisfy the equation [5] as perfectly as possible it is proposed here to estimate the PV by solving the following constrained optimization problem:

subject to:

$$
\min \sum_{i=1}^{n}\left(\exp \left(\sum_{j=1}^{n} a_{i j} w_{j}-n w_{i}\right)-1\right)^{2}
$$

$$
\sum_{i=1}^{n} w_{i}=1, \quad w_{i} \geq 0, \quad i, j=1, \ldots, n
$$

Let it be given the above proposed method of PVs deriving the name Exponential Quotient Squared Deviations Minimization Method (denoted EQSDM). On the contrary to the EM this method proposed does not suffer from drawbacks already mentioned. It can be easily applied to reciprocal and nonreciprocal matrices as well. The computations performed during PVs deriving procedure are considerably easier than in the case of EM and, what is more, they can be easily performed with the application of standard office software packages commonly available. Additionally, what is quite important, EQSDM holds the condition of order preservation.

\section{An Example Scenario Based Analysis}

Hereby, it is provided the EQSDM efficacy analysis on the basis of already published case study. It is shown that EQSDM preserve the intensity of preferences in the case where EM fails.

First, however it must be clarified the meaning of order preservation condition formulated by Bana e Costa et. al. (2008). They provide the following definition: for all alternatives a1, $\mathrm{a} 2$, a3, a4 such that a1 dominates a 2 and a3 dominates a4, and the extent to which a1 dominates $\mathrm{a} 2$ is greater than the extent to which a 3 dominates a4, one has not only w1 $>\mathrm{w} 2$ and $w 3>\mathrm{w} 4$ but also $\mathrm{w} 1 / \mathrm{w} 2>\mathrm{w} 3 / \mathrm{w} 4$ for derived PV.

Now it will be analyzed the scenario provided in (Bana e Costa et. al. 2008) to verify the efficacy of EQSDM. Let the PCM be as follows:

$$
\left[\begin{array}{ccccc}
1 & 2 & 3 & 5 & 9 \\
1 / 2 & 1 & 2 & 4 & 9 \\
1 / 3 & 1 / 2 & 1 & 2 & 8 \\
1 / 5 & 1 / 4 & 1 / 2 & 1 & 7 \\
1 / 9 & 1 / 9 & 1 / 8 & 1 / 7 & 1
\end{array}\right]
$$


Pawel Tadeusz KAZIBUDZKI. An Exponential Utility Approach to Eigenvector Method in the Analytic Hierarchy Process: An Idea Intro

As it can be seen here according to a common linguistic interpretation for AHP, a1 strongly dominates a4 (a1/a4=5), and a4 very strongly dominates a5 (a4/a5=7). That implies a1/ a4<a4/a5. However the PV derived from EM provides [0.4262, 0.2809, 0.1652, 0.1008, 0.0269] ${ }^{\mathrm{T}}$ and yields the ratios a1/a4=4.218 $>\mathrm{a} 4 / \mathrm{a} 5=3.741$ which violate the COP.

Let it now be applied the new proposed method EQSDM. The PV derived from EQSDM provides $[0.434659,0.282449,0.163602,0.097671,0.021620]^{\mathrm{T}}$ and yields the ratios $\mathrm{a} 1 / \mathrm{a} 4=4.450246<\mathrm{a} 4 / \mathrm{a} 5=4.517675$ which, on the contrary to EM, satisfy the COP. The research findings will be concluded now with the following statement: exponential quotient squared deviations minimization method is probably as good as eigenvalue method to uniquely capture the ratio scale rank order inherent in inconsistent pairwise comparison judgments and sometimes it is even better.

\section{Conclusion}

To summarize, there is at least one valid method for deriving the priority vector from a pairwise comparison matrix, particularly when the matrix is inconsistent, which is equally satisfying as eigenvalue method and sometimes it is even better. It is so because this method can be applied to both reciprocal and nonreciprocal matrices, it is computationally simpler and what is most important it does not violate condition of order preservation. As indicated, there is at least one such method, named here: exponential quotient squared deviations minimization method. Obviously, it needs further studies and analysis to be strengthen, especially from the viewpoint of the statement provided above. However, this process has been already initiated by the author of this article, and its findings will hopefully be published very soon as well.

\section{Acknowledgements}

I owe Andrzej Z. Grzybowski my thanks for the courtesy of presenting his relatively novel approach to estimating priority weights via personal communication, as well for the privilege to study his yet unpublished paper, entitled: A note on some optimization based methods for estimating priority weights.

\section{References}

Bana e Costa, C. A., Vansnick, J. C. (2008). A critical analysis of the eigenvalue method used to derive priorities in AHP. European Journal of Operational Research, Vol. 187, p. 1422-1428.

Basak, I. (1998). Comparison of statistical procedures in analytic hierarchy process using a ranking test. Mathematical Computation Modeling, Vol. 28, p. 105-118.

Bryson, N. (1995). A goal programming method for generating priority vectors. Journal of the Operational Research Society, Vol. 46, p. 641-648.

Budescu, D. V., Zwick, R., Rapoport, A. (1986). Comparison of the analytic hierarchy process and the geometric mean procedure for ratio scaling. Applied Psychological Measurement, Vol. 10, p. 69-78.

Choo, E. U., Wedley, W. C. (2004). A common framework for deriving preference values from pairwise comparison matrices. Computers \& Operations Research, Vol. 31, p. 893-908.

Cook, W. D., Kress, M. (1988). Deriving weights from pairwise comparison ratio matrices: An axiomatic approach. European Journal of Operational Research, Vol. 37, p. 355-362.

Crawford, G., Williams, C. A. (1985). A note on the analysis of subjective judgment matrices. Journal of Mathematical Psychology, Vol. 29, p. 387-405.

\author{
PROBLEMS \\ OF MANAGEMENT \\ IN THE $21{ }^{\text {st }}$ CENTURY \\ Volume 1, 2011 \\ 65
}


PROBLEMS

OF MANAGEMENT

IN THE $21^{\text {st }}$ CENTURY

Volume 1, 2011

66 Crawford, G. B. (1987). The geometric mean procedure for estimating the scale of a judgment matrix.

Mathematical Modeling, Vol. 9, No. 3-5, p. 327-334.

Dong, Y., Xu, Y., Li, H., Dai, M. (2008). A comparative study of the numerical scales and the prioritization methods in AHP. European Journal of Operational Research, Vol. 186, p. 229-242.

Fichtner, J. (1986). On deriving priority vectors from matrices of pairwise comparisons. Socio-Economic Planning Science, Vol. 20, Issue 6, p. 341-345.

Grzybowski, A. Z. (2010). Goal programming approach for deriving priority vectors - some new ideas. Scientific Research of the Institute of Mathematics and Computer Science, Vol. 1(9), p. 17-27.

Hashimoto, A. (1994). A note on deriving weights from pairwise comparison ratio matrices. European Journal of Operational Research, Vol. 73, Issue 1, p. 144-149.

Hovanov, N. V., Kolari, J. W., Sokolov, M. V. (2008). Deriving weights from general pairwise comparison matrices. Mathematical Social Sciences, Vol. 55, Issue 2, p. 205-220.

Lin, C.-C. (2006). An enhanced goal programming method for generating priority vectors. Journal of the Operational Research Society, Vol. 57, p. 1491-1496.

Lipovetsky, S., Tishler, A. (1997). Interval estimation of priorities in the AHP. European Journal of Operational Research, Vol. 114, p. 153-164.

Saaty, T. L. (1994). Fundamentals of the Analytic Hierarchy Process. RWS Publications, Pittsburg, PA.

Saaty, T. L., Hu, G. (1998). Ranking by Eigenvector versus other methods in the Analytic Hierarchy Process. Applied Mathematics Letters, Vol. 11, Issue 4, p. 121-125.

Saaty, T. L. (1980). The Analytic Hierarchy Process. McGraw Hill, New York.

Saaty, T. L., Vargas, L. G. (1984). Comparison of eigenvalue, logarithmic least square and least square methods in estimating ratio. Journal of Mathematical Modeling, Vol. 5, Issue 5, p. 309-324.

Srdjevic, B. (2005). Combining different prioritization methods in the analytic hierarchy process synthesis. Computers \& Operations Research, Vol. 32, p. 1897-1919.

Sun, L., Greenberg, B. S. (2006). Multiple group decision making: optimal priority synthesis from pairwise comparisons. Journal of Optimization Theory and Applications, Vol. 130, No. 2, p. 317-338.

Advised by Andrzej Z. Grybowski, Technical University of Czestochowa, Poland

Received: May 02, 2011

Accepted: May 30, 2011

Pawel Tadeusz Kazibudzki Dr., M.B.A., Assistant Professor, The Faculty of Social Sciences, Jan Dlugosz University in Czestochowa, Waszyngtona 4/8, 42-200, Czestochowa, Poland.

E-mail: emailpoczta@gmail.com

Website: http://www.ajd.czest.pl/p993,Kazibudzki 